\title{
Tecnologias Aplicadas ao Ensino e Aprendizagem com LIBRAS: Um Mapeamento Sistemático
}

\author{
Venilton FalvoJr ${ }^{1}$, Catherine Martins Falvo ${ }^{2}$, \\ Lilian Passos Scatalon ${ }^{1}$, Ellen Francine Barbosa ${ }^{1}$ \\ ${ }^{1}$ Instituto de Ciências Matemáticas e de Computação (ICMC) \\ Universidade de São Paulo (USP) - São Carlos, SP, Brasil \\ \{falvojr,lilian.scatalon\}@usp.br, francinedicmc.usp.br \\ ${ }^{2}$ Universidade de Araraquara (UNIARA) - Araraquara, SP, Brasil \\ cattmartins@gmail.com
}

\begin{abstract}
Considering the last census, it is estimated that Brazil has 9.7 million people with hearing impairment (about 5.1\% of the population). These people are potential users of the Brazilian Sign Language (LIBRAS), but only a portion of them will have access to literacy, due to national socioeconomic difficulties. On the other hand, the advent of technology has had a positive impact on teaching and learning dynamics. In this context, this work aims to obtain an overview of the technological solutions that have been used in the teaching of sign languages, with an emphasis on LIBRAS. To this end, we conducted a Systematic Mapping (SM), whose stage of manual search identified 46 primary studies in Brazilian conferences, journals and magazines. In general, it results indicate that the technologies have already been consistently contributing to the teaching of LIBRAS. Most studies have been published in the past 10 years, which suggests an emerging line of research. In this sense, different types of approaches were identified, from analysis and design to the construction and validation of technological solutions.
\end{abstract}

Resumo. Considerando o último censo, estima-se que o Brasil tenha 9,7 milhões de pessoas com deficiência auditiva (cerca de 5,1\% da população), as quais têm como principal alternativa para comunicação a Lingua Brasileira de Sinais (LIBRAS). Entretanto, apenas uma parcela dessas pessoas terá acesso à alfabetização, tendo em vista as dificuldades socioeconômicas nacionais. Por outro lado, o advento da tecnologia vem impactando positivamente nas dinâmicas de ensino e aprendizagem. Nesse contexto, este trabalho tem como objetivo obter uma visão geral das soluções tecnológicas que vêm sendo utilizadas no ensino baseado em línguas de sinais, com ênfase na LIBRAS. Para isso, um Mapeamento Sistemático (MS) foi conduzido, cuja etapa de busca manual identificou 46 estudos primários em conferências, periódicos e revistas brasileiras. De modo geral, observou-se que as tecnologias já vêm contribuindo consistentemente para o ensino através da LIBRAS. A maioria dos estudos foram publicados nos últimos 10 anos, o que sugere uma linha de pesquisa emergente. Nesse sentido, foram identificadas diferentes tipos de abordagens, desde a análise e projeto até a construção e validação de soluções tecnológicas. 
IX Congresso Brasileiro de Informática na Educação (CBIE 2020)

Anais do XXXI Simpósio Brasileiro de Informática na Educação (SBIE 2020)

\section{Introdução}

A maioria dos idiomas são baseados em sons audíveis. Sendo assim, as pessoas estão acostumadas à linguagem falada e, para a maioria delas, a comunicação é regida pela audição [Duke 2009]. No entanto, existem 466 milhões de pessoas, 6,1\% da população mundial, com perda auditiva incapacitante [WHO 2018]. Com isso, grande parte delas têm a necessidade de "ouvir com os olhos", algo possível por meio das línguas de sinais.

As línguas de sinais são formas de comunicação essencialmente baseadas no sentido da visão, nas quais as informações transmitidas visualmente usando diferentes formas/movimentos das mãos. Para isso, seus usuários combinam movimentos articulados das mãos, expressões faciais, movimentos da cabeça e do corpo para comunicar sentimentos, intenções, humor, além de ideias complexas ou abstratas [Duke 2009].

No Brasil, a Língua Brasileira de Sinais (LIBRAS) foi reconhecida oficialmente em 2002. Entretanto, desde então poucas iniciativas vêm sendo fomentadas em âmbito nacional para inclusão social de seus usuários. Segundo o último censo demográfico do Instituto Brasileiro de Geografia e Estatística (IBGE) [IBGE 2010], existem cerca de 9,7 milhões de pessoas com deficiência auditiva no Brasil, o que equivale a $5,1 \%$ da população. Infelizmente nem todos esses usuários em potencial serão alfabetizados na LIBRAS, tendo em vista as dificuldades socioeconômicas do país.

Por outro lado, o advento das Tecnologias de Informação e Comunicação (TICs) tem modificado desde as interações sociais entre as pessoas até as práticas de ensino e aprendizagem no contexto educacional. De fato, a educação é um processo contínuo de desenvolvimento para que o aprendiz se integre na sociedade ou grupo ao qual pertence [Giordano et al. 2017]. Logo, a inclusão social/digital destaca-se no processo educacional moderno, no qual as TICs podem ser ferramentas essenciais.

Sendo assim, para o desenvolvimento de ambientes educacionais eficazes, é fundamental que sejam consideradas as características intrínsecas dos aprendizes, incluindo suas necessidades especiais. No entanto, pessoas com deficiência auditiva ainda enfrentam dificuldades no processo de ensino devido a ambientes inadequados de aprendizagem, que geralmente não são desenvolvidos com suporte a línguas de sinais [Snoddon 2018].

É necessário, portanto, refletir sobre o ensino e aprendizagem das línguas de sinais, considerando o crescente uso de tecnologias no domínio educacional. De fato, as TICs podem facilitar o acesso ao conhecimento, especialmente no contexto de pessoas com algum tipo de deficiência.

Nesse cenário, este artigo apresenta uma visão geral sobre o uso da LIBRAS na educação através das TICs. Nesse contexto, um Mapeamento Sistemático (MS) foi conduzido, inicialmente com ênfase em sua busca automatizada [Falvojr et al. 2020]. De modo complementar, neste trabalho a busca manual tem destaque, evidenciando os resultados em âmbito nacional. Trata-se de 46 estudos, sobretudo com foco na LIBRAS.

Este artigo está organizado da seguinte forma: a Seção 2 descreve o MS e como a busca manual foi conduzida. A Seção 3 apresenta os resultados obtidos em âmbito nacional, além de relacioná-los às questões de pesquisa do MS. A discussão a respeito dos trabalhos identificados é apresentada na Seção 4. Finalmente, as principais conclusões e perspectivas de trabalhos futuros são sumarizadas na Seção 5. 
IX Congresso Brasileiro de Informática na Educação (CBIE 2020)

Anais do XXXI Simpósio Brasileiro de Informática na Educação (SBIE 2020)

\section{Método}

Em um estudo anterior foi realizado um MS sobre ensino baseado em línguas de sinais mediante o uso de tecnologia [Falvojr et al. 2020], resultando em um total de 139 estudos primários selecionados a partir da busca automatizada em bases de dados internacionais.

Este trabalho seguiu o mesmo protocolo, com base em diretrizes consolidadas [Zhang et al. 2011, Petersen et al. 2015, Kitchenham and Charters 2007], mas tendo como foco conferências e periódicos nacionais. A seguir são sumarizadas as principais etapas conduzidas.

\subsection{Questões de Pesquisa}

O objetivo do MS foi determinar como a tecnologia está sendo aplicada no ensino e aprendizagem de línguas de sinais, o que derivou as seguintes Questões de Pesquisa (QP) [Falvojr et al. 2020]:

- QP1: Quais tipos de soluções tecnológicas vêm sendo empregadas no ensino com línguas de sinais?

- QP2: Qual conteúdo tem sido ensinado por meio dessas tecnologias?

- QP3: Além da LIBRAS, quais outras línguas de sinais têm sido utilizadas?

\subsection{Critérios de Seleção}

Foram definidos os seguintes critérios de seleção para o MS [Falvojr et al. 2020]:

- Critério de Inclusão:

- Os estudos apresentam contribuições (software ou hardware ou teóricas) para o ensino e a aprendizagem com línguas de sinais.

- Critérios de Exclusão:

- Estudos que não foram publicados entre 2000 e 2019;

- Estudos classificados como resumos, resumos de conferências/editoriais, literatura cinza ou capítulos de livros;

- Estudos não apresentados em inglês ou português;

- Estudos não acessíveis em texto completo;

- Estudos duplicados ou superficialmente complementares a outros.

\subsection{Busca Manual}

Para a etapa de busca manual, foram selecionados eventos nacionais especializados em educação, os quais foram definidos com o apoio de especialistas no domínio em questão.

Nesse contexto, oito conferências/periódicos/revistas foram definidos durante a condução da busca manual nacional. Nesta etapa, título, resumo e palavras-chave foram essenciais na avaliação preliminar dos estudos. Como resultado, 82 estudos foram pré-selecionados e avaliados por meio de uma dupla checagem, conduzida por dois dos autores deste trabalho. Assim, todas as divergências de classificação entre os autores (baseadas nos critérios de seleção) foram analisadas e consentidas individualmente. Por fim, a leitura dos estudos na íntegra foi conduzida para uma classificação mais eficaz dos estudos selecionados.

Com isso, 46 publicações nacionais foram classificadas como relevantes, as quais serão analisadas nas seções 3 e 4 deste artigo. A Tabela 1 apresenta as conferências e periódicos utilizados, além de seus respectivos estudos primários. Como resultado, foi possível obter uma visão ampla com relação a LIBRAS, tema onipresente nas publicações em questão. 
IX Congresso Brasileiro de Informática na Educação (CBIE 2020)

Anais do XXXI Simpósio Brasileiro de Informática na Educação (SBIE 2020)

Tabela 1. Busca manual nacional

\begin{tabular}{llll}
\hline Conferências/Periódicos & Fonte & Pré-selecionados & Selecionados \\
\hline DesafIE & CEIE & 0 & - \\
JAIE & CEIE & 0 & - \\
RBIE & CEIE & 5 & 2 \\
RENOTE & CINTED/UFRGS & 21 & 13 \\
SBIE & CEIE & 31 & 13 \\
WAVE2 & CEIE & 0 & - \\
WCBIE & CEIE & 16 & 11 \\
WIE & CEIE & 9 & 7 \\
Total & & $\mathbf{8 2}$ & $\mathbf{4 6}$ \\
\hline
\end{tabular}

\subsection{Extração de Dados}

Devido ao grande número de estudos selecionados na busca manual, optou-se por não incluir todas as referências neste trabalho. Sendo assim, o formulário de extração de dados e as respectivas informações extraídas para cada artigo estão disponíveis online ${ }^{1}$.

De modo geral, um total de 185 estudos primários foram identificados pelo MS, dos quais 139 já foram discutidos em um estudo anterior [Falvojr et al. 2020]. Com isso, apenas os estudos obtidos no Brasil serão considerados neste trabalho. Sendo assim, os 46 estudos primários selecionados por meio da busca manual nacional foram devidamente identificados pelos prefixos $B R A$ e serão analisados e discutidos nas seções a seguir.

\section{Resultados}

Considerando os 46 estudos selecionados, nesta seção cada questão de pesquisa será abordada, evidenciando suas respectivas constatações. Levando em conta a quantidade de publicações nacionais por ano, foi possível identificar uma linha de tendência linear crescente (Figura 1). Portanto, é estatisticamente possível que este domínio de pesquisa esteja em ascensão no Brasil. Além disso, o número reduzido de publicações anteriores a 2010 (cerca de 10\%) pode ser considerado para a definição de critérios de exclusão mais rigorosos em uma replicação ou atualização deste MS.

\subsection{Soluções Tecnológicas (QP1)}

A primeira questão de pesquisa tem como objetivo identificar os tópicos formais de desenvolvimento que vêm sendo investigados no contexto educacional por meio das línguas de sinais. As áreas identificadas foram classificadas com base na estrutura Software Engineering Body of Knowledge (SWEBOK) [Bourque et al. 2014, Petersen et al. 2015]. Sendo assim, os estudos primários nacionais foram classificados nas seguintes áreas:

- Construção de Software: trabalhos com ênfase no desenvolvimento de soluções;

- Projeto de Software: publicações com foco na análise e projeto de soluções;

- Fundamentos da Engenharia: estudos com ênfase em avaliações empíricas (survey, estudo de caso ou experimento [Wohlin et al. 2012]);

- Qualidade de Software: trabalhos pautados por critérios de qualidade técnicos.

\footnotetext{
${ }^{1}$ https://bit.ly/SBIE20-SM-DataExtraction
} 


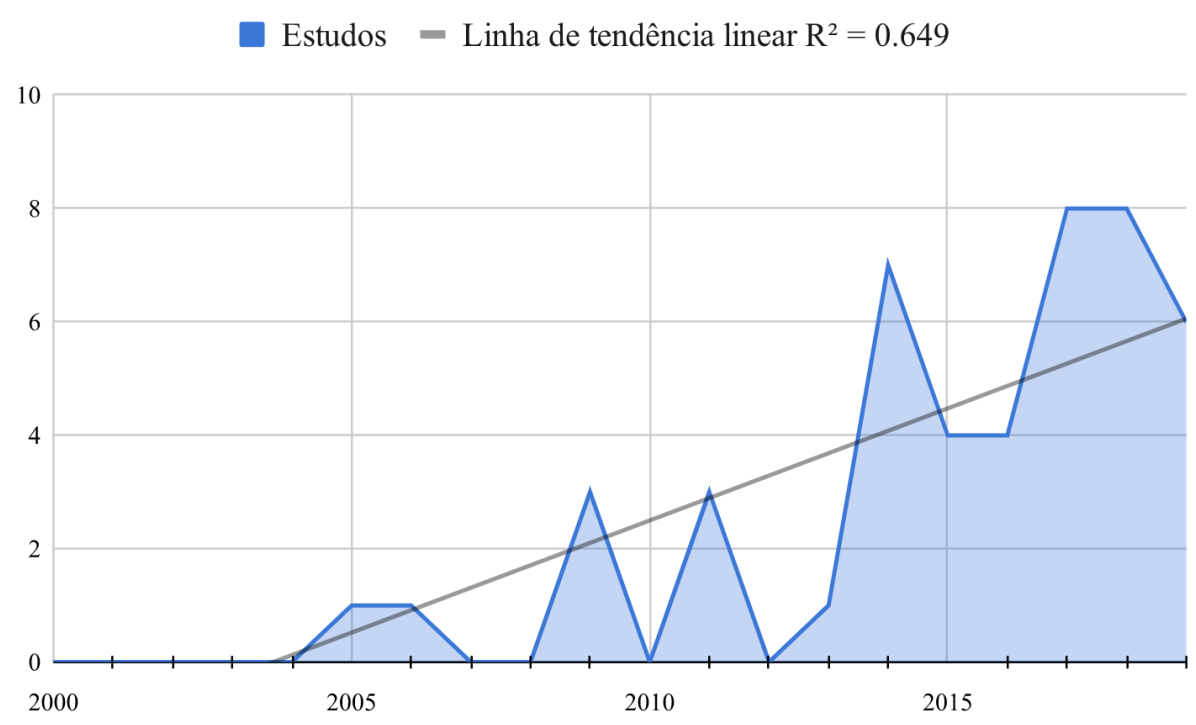

Figura 1. Linha de tendência linear $\left(R^{2}\right)$ de publicações nacionais por ano.

Cerca de 50\% dos estudos primários nacionais têm como contribuição principal a construção de soluções que exploram as vertentes de software ou hardware. Sendo assim, tais estudos foram classificados na área de "Construção de Software" (BRA1, BRA2, BRA3, BRA4, BRA5, BRA12, BRA15, BRA19, BRA20, BRA21, BRA23, BRA24, BRA25, BRA29, BRA31, BRA33, BRA34, BRA36, BRA39, BRA41, BRA42, BRA43, BRA45). Em menor proporção, o equivalente a $5 \%$ dos resultados foram rotulados como "Projeto de Software" (BRA13, BRA30, BRA32, BRA38, BRA44).

Por outro lado, existe uma quantidade expressiva de estudos baseados em "Fundamentos da Engenharia", ou seja, que possuem avaliações empíricas. Em números, tais publicações equivalem a 19\% dos estudos primários, o que mostra a importância das avaliações formais (BRA14, BRA17, BRA22, BRA27, BRA28, BRA35, BRA37, BRA40, BRA46).

Entretanto, mais artigos possuem avaliações empíricas, mas foram classificados em outras áreas, semanticamente adequadas a suas respectivas contribuições primárias. Nesse cenário, $52.17 \%$ dos estudos primários nacionais apresentam algum tipo de avaliação empírica. Desse modo, é possível aferir que aproximadamente a metade dos estudos primários identificados possuem algum tipo de avaliação formal.

Por fim, existe um número relevante de estudos com ênfase em validações e/ou avaliações não empíricas, classificadas na área de "Qualidade de Software". Nesse contexto, a busca manual nacional obteve $19 \%$ de seus estudos nesta categoria (BRA6, BRA7, BRA8, BRA9, BRA10, BRA11, BRA16, BRA18, BRA26), evidenciando que esta é uma área muito investigada no Brasil.

De modo geral, considerando os tipos de solução: $58,7 \%$ dos estudos primários têm ênfase em software, $34,8 \%$ são contribuições teóricas e 6,5\% têm foco em hardware. Desta forma, é possível concluir que a maioria das soluções tecnológicas para ensino e aprendizagem de línguas de sinais vêm se baseando em software, em âmbito nacional. Neste contexto, cada um dos estudos foi categorizado mais especificamente com relação a solução proposta (Figura 2). 


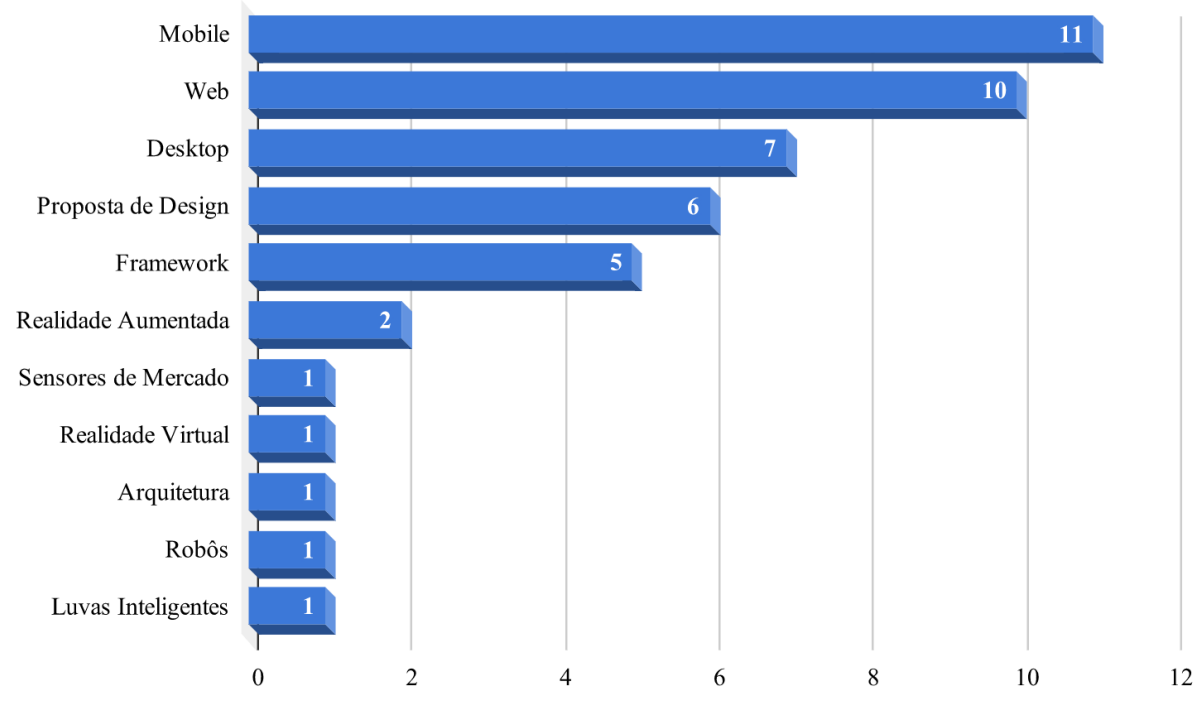

Figura 2. Publicações por tipo de solução específica.

Tais informações evidenciam a dominância de algumas plataformas de desenvolvimento de software: Mobile (BRA2, BRA6, BRA7, BRA8, BRA10, BRA11, BRA16, BRA18, BRA33, BRA34, BRA36); Web (BRA3, BRA5, BRA9, BRA12, BRA19, BRA20, BRA21, BRA39, BRA42, BRA43); e Desktop (BRA4, BRA15, BRA23, BRA24, BRA40, BRA41, $B R A 46)$. Juntas, essas plataformas equivalem a mais de $60 \%$ dos estudos primários nacionais. No entanto, não foram identificadas soluções explorando o conceito multiplataforma, como o desenvolvimento de soluções híbridas. Essa constatação pode denotar uma lacuna relevante em termos de portabilidade, já que a falta de uma experiência unificada pode prejudicar e/ou limitar a experiência dos usuários brasileiros.

Sob outra perspectiva, diversas propostas de análise e projeto foram identificadas (BRA13, BRA17, BRA30, BRA32, BRA37, BRA38), mostrando que as potenciais soluções vêm sendo avaliadas pela comunidade científica antes da sua efetiva implementação.

Por fim, os estudos primários demonstram que algumas técnicas e tecnologias vêm ganhando notoriedade. Nesse sentido, merece destaque o uso dos conceitos de Realidade Aumentada (RA) e Realidade Virtual (RV) (BRA25, BRA29, BRA31). Além disso, contribuições baseadas em API, frameworks e arquiteturas de software também foram propostas (BRA22, BRA27, BRA28, BRA35, BRA44, BRA45), mostrando que existem algumas iniciativas para a criação de soluções mais genéricas e estruturadas nesse domínio.

\subsection{Tópicos Educacionais (QP2)}

A segunda QP diz respeito aos tópicos educacionais das publicações, com o objetivo de analisar o público-alvo e o contexto educacional das soluções propostas. Para isso, foram extraídos os temas relacionados ao ensino e aprendizagem, tendo em vista os estudos relevantes deste trabalho. A Tabela 2 sintetiza todos os tópicos educacionais identificados, considerando a busca manual nacional.

Cerca de $41,3 \%$ dos estudos exploram o ensino de LIBRAS, evidenciando que ainda existem muitos desafios educacionais sendo investigados pela comunidade científica. Por outro lado, 21,7\% são aplicados à educação sem um tópico de ensino específico, em geral essas soluções tendem a ser mais flexíveis e disruptivas. 
IX Congresso Brasileiro de Informática na Educação (CBIE 2020)

Anais do XXXI Simpósio Brasileiro de Informática na Educação (SBIE 2020)

Tabela 2. Tópicos Educacionais

\begin{tabular}{lll}
\hline Tópicos Educacionais & Estudos & \% \\
\hline Línguas de Sinais & 19 & $41,3 \%$ \\
Geral & 10 & $21,7 \%$ \\
Português (Letramento Bilíngue) & 9 & $19,6 \%$ \\
Ciência da Computação & 4 & $8,7 \%$ \\
Língua de Sinais Escrita & 3 & $6,5 \%$ \\
Alfabeto & 1 & $2,2 \%$ \\
Total & $\mathbf{4 6}$ & $\mathbf{1 0 0 \%}$ \\
\hline
\end{tabular}

Outro tópico que merece destaque diz respeito ao ensino do Português (BRA5, BRA13, BRA15, BRA17, BRA21, BRA25, BRA26, BRA40, BRA41), tendo em vista a grande importância do letramento bilíngue para a inclusão social dos usuários das línguas de sinais. Particularmente, os trabalhos brasileiros se destacaram pelos esforços para o letramento bilíngue Português/LIBRAS, que representam quase $20 \%$ dos estudos primários. Essa preocupação é extremamente relevante para que pessoas com deficiência auditiva possam expandir sua capacidade de comunicação através da sua língua nativa.

Os outros $17,4 \%$ estão distribuídos nos seguintes tópicos de ensino: Ciência da Computação (BRA14, BRA33, BRA43, BRA45); Língua de Sinais Escrita (BRA3, BRA19, $B R A 20)$, no qual destaca-se o SignWriting ${ }^{2}$; e Alfabeto (BRA24).

Ademais, identificou-se o público-alvo dos estudos, com a intenção de complementar a resposta desta QP. Assim, a maioria (aproximadamente 76,1\%) tem relação direta somente com usuários surdos, dos quais cerca de $20 \%$ têm como foco principal em crianças (BRA1, BRA3, BRA5, BRA6, BRA24, BRA25, BRA37, BRA39, BRA41). Isso evidencia que existem muitas iniciativas para inclusão social de surdos e/ou pessoas com deficiência auditiva, em diferentes contextos.

Em contrapartida, 24,9\% dos estudos têm um público-alvo mais abrangente, investigando formas de reduzir as barreiras entre usuários e não usuários das línguas de sinais (BRA2, BRA7, BRA8, BRA10, BRA11, BRA12, BRA16, BRA18, BRA22, BRA27, $B R A 35)$. Por conta disso, tais estudos tendem a investigar as línguas de sinais de maneira secundária, mas como uma ferramenta essencial de ensino e inclusão social.

\subsection{Línguas de Sinais (QP3)}

Analisando o histórico de publicações nacionais (Figura 1), a LIBRAS possui apenas cinco estudos anteriores a 2010 (BRA3, BRA15, BRA26, BRA41, BRA42), aproximadamente $11,3 \%$ de um total de 44 estudos primários nessa língua de sinais. Isso sugere um aumento expressivo das publicações relacionadas à LIBRAS, em especial nos últimos dez anos. Sendo assim, o baixo índice de estudos relevantes anteriores a 2010 pode pautar os critérios de seleção em uma replicação ou estudo futuro.

Por fim, a SignWriting aparece com duas contribuições, mostrando que a língua de sinais escrita também possui apelo cientifico nacionalmente (BRA3, BRA20). Por outro lado, nenhum dos estudos primários nacionais investigam múltiplas línguas de sinais. Por-

\footnotetext{
${ }^{2}$ Sistema de escrita que usa símbolos visuais para representar as formas das mãos, movimentos e expressões faciais das línguas de sinais. Mais informações em https://signwriting. org.
} 
tanto, nenhum trabalho apresenta uma proposta genérica ou com alto nível de abstração para o desenvolvimento soluções baseadas em múltiplas línguas de sinais, algo bastante relevante para criação de ambientes educacionais de escala global.

É possível concluir que existem muitas pesquisas interessantes no que tange ao domínio investigado neste MS. Ainda assim, a maioria das soluções são construídas sem padrões e/ou estilos arquiteturais, o que dificulta o acesso à informação e criação de soluções mais inteligentes e colaborativas. Na próxima seção este assunto será discutido, juntamente com outros temas relevantes, entre os quais a LIBRAS será o tema central.

\section{Discussão}

Com base nos resultados apresentados, é possível concluir que a LIBRAS, no domínio educacional, vem sendo consistentemente apoiada por soluções tecnológicas. Sendo assim, estudos nos quais a LIBRAS é a língua de sinais primária somam 44 publicações, obtidas por meio da busca manual nacional. Alguns desses estudos primários são discutidos a seguir com a intenção de sintetizar suas principais contribuições.

Soluções baseadas em avatares equivalem a $20 \%$ das contribuições (BRA6, BRA7, BRA8, BRA10, BRA11, BRA16, BRA18, BRA29, BRA33). Portanto, é possível inferir que avatares $3 \mathrm{D}$ representam parte significativa do estado da prática na representação das línguas de sinais. Em particular, boa parte dos estudos investiga a efetividade desse tipo de solução, principalmente por meio da comparação de aplicativos educacionais móveis (BRA6, BRA7, BRA8, BRA10, BRA11, BRA16, BRA18). Dentre os mais avaliados estão: Hand Talk ${ }^{3}$; ProDeaf (descontinuado, devido a "fusão" com o Hand Talk); VLibras ${ }^{4}$; e Rybená $a^{5}$. Considerando os resultados apresentados, o Hand Talk se mostrou a frente dos concorrentes, saindo-se melhor na interpretação, tradução e eventuais desambiguações.

Vários estudos selecionados investigam conceitos de gamificação em suas soluções educacionais (BRA2, BRA12, BRA23, BRA24, BRA25, BRA31, BRA34, $B R A 36, B R A 37, B R A 39)$, principalmente os destinados ao público infantil. Nesse sentido, [Resende et al. 2019] descrevem o desenvolvimento de um jogo chamado $\mathrm{Li}$ brasBot, desenvolvido para ser um Recurso Educacional Aberto (REA) multidisciplinar. [Lopes et al. 2018] apresentam o aplicativo LibrAR que implementa os conceitos de RA/RV para auxiliar no ensino das letras e números em LIBRAS. Por fim, [Vale et al. 2018] apresentam o projeto e construção do jogo Gestus, que tem por objetivo ensinar LIBRAS para crianças de forma lúdica. Dentre os jogos identificados, o Gestus é o que possui a interface visual mais elaborada e amigável.

Finalmente, algumas publicações contribuíram na vertente teórica por meio do conceito de Arquiteturas Pedagógicas (AP) (BRA22, BRA27, BRA35). Segundo [Reinoso et al. 2017], uma AP pode ser definida pelo seguinte conjunto dos componentes: (i) objetivo de aprendizagem - o que aprender; (ii) atividades - o que fazer; (iii) método - como desenvolver as atividades; e (vi) recursos digitais - com quais ferramentas. Em outras palavras, são estruturas de aprendizagem compostas pela abordagem pedagógica, software, Internet, IA, Educação a Distância (EAD) e concepção de tempo e espaço [Tavares et al. 2017].

\footnotetext{
${ }^{3}$ https: / / handtalk.me

${ }^{4}$ https://vlibras.gov.br

${ }^{5}$ https://portal.rybena.com.br/site-rybena
} 
De modo geral, observou-se uma diversidade interessante de soluções neste MS. Com isso, foi possível identificar o uso massivo das TICs, além de novas técnicas pedagógicas e abordagens de desenvolvimento bastante sofisticadas. Por outro lado, poucos estudos contribuíram no sentido de disponibilizar um arcabouço público visando o desenvolvimento canônico e colaborativo de soluções educacionais voltadas às línguas de sinais. Sendo assim, acredita-se que padrões de projeto e desenvolvimento possam ajudar na proposição de aplicações educacionais com uma arquitetura genérica, tendo em vista soluções mais flexíveis de ensino e aprendizagem com línguas de sinais.

\section{Conclusões e Trabalhos Futuros}

Neste artigo, foi apresentada uma visão geral das pesquisas realizadas sobre o uso da tecnologia para o ensino e aprendizagem através das línguas de sinais, com foco na LIBRAS. Um MS foi conduzido previamente por [Falvojr et al. 2020], cuja busca manual nacional identificou 46 estudos primários, os quais são o foco deste trabalho. Com isso, os artigos foram classificados de acordo com suas soluções tecnológicas, tópicos educacionais e línguas de sinais para responder as QP definidas.

Em síntese, identificou-se que a maioria das soluções dizem respeito ao design ou construção de software. Adicionalmente, metade dos estudos primários apresentam alguma avaliação empírica, o que evidencia a importância de um processo de avaliação formal. Em aspectos técnicos, as soluções identificadas com foco no ensino e aprendizagem de LIBRAS estão divididas entre as plataformas Web, Mobile e Desktop. No entanto, alguns estudos enfatizam abordagens mais específicas como tradução de máquina, sensores, RA/RV, frameworks, arquiteturas e, em menor grau, robótica.

Sobre os tópicos educacionais, existe uma grande incidência de soluções educacionais focadas no ensino de LIBRAS. Além disso, abordagens de ensino inclusivas e iniciativas de letramento bilíngue também têm o devido destaque. Tais iniciativas são essenciais para a inclusão social dos usuários da LIBRAS, que podem expandir suas vias de comunicação através da língua escrita do seu país.

Como trabalhos futuros, pretende-se investigar detalhadamente os aspectos estruturais e técnicos para definição de uma arquitetura de referência para aplicações educacionais baseadas em línguas de sinais. Em especial, o conceito de REA, apesar do pouco destaque neste MS, poderia derivar soluções interessantes, principalmente tendo em vista a criação de uma interface computacional pública e colaborativa para o ensino/aprendizagem através das línguas de sinais. Por fim, a condução de uma Revisão Sistemática será avaliada, com a intenção de refinar o escopo do mapeamento atual e evidenciar novas lacunas de pesquisa.

\section{Agradecimentos}

O presente trabalho foi realizado com apoio da Fundação de Amparo à Pesquisa do Estado de São Paulo (FAPESP) - \#2018/26636-2; Coordenação de Aperfeiçoamento de Pessoal de Nível Superior - Brasil (CAPES) - Código Financeiro 001; e CNPq.

\section{Referências}

Bourque, P., Fairley, R. E., and Society, I. C. (2014). Guide to the Software Engineering Body of Knowledge (SWEBOK(R)): Version 3.0. IEEE Computer Society Press, Washington, DC, USA, 3rd edition. 
IX Congresso Brasileiro de Informática na Educação (CBIE 2020)

Anais do XXXI Simpósio Brasileiro de Informática na Educação (SBIE 2020)

Duke, I. (2009). The Everything Sign Language Book: American Sign Language Made Easy. Adams Media Corporation, Avon, MA.

Falvojr, V., Scatalon, L. P., and Barbosa, E. F. (2020). The role of technology to teaching and learning sign languages: A systematic mapping. In 2020 IEEE Frontiers in Education Conference (FIE), pages 1-9.

Giordano, C. V., Langhi, C., de Albergaria Henriques da Silva, A. S., Croce, E. F., de Oliveira, G. B., Domingues, I., da Silveira Pinto, L., Matos, S., and Cilli, T. (2017). A Tecnologia Da Informação e Comunicação Nas Práticas Educacionais. Independently Published, São Paulo, SP.

IBGE (2010). Conheça o brasil - população: Pessoas com deficiência. https://bit.ly/2Zf791V. Accessed: 2020-07-10.

Kitchenham, B. and Charters, S. (2007). Guidelines for performing systematic literature reviews in software engineering.

Lopes, M., Silva, L., and Reis, D. (2018). Librar: aplicativo de aprendizagem de libras usando realidade aumentada e realidade virtual em dispositivo móvel. Anais dos Workshops do Congresso Brasileiro de Informática na Educação (WCBIE), 1(1):10.

Petersen, K., Vakkalanka, S., and Kuzniarz, L. (2015). Guidelines for conducting systematic mapping studies in software engineering: An update. Information and Software Technology, 64:1-18.

Reinoso, L., Amorim, M., Tavares, O., and Almeida, R. (2017). Framework cap 1.0 para criação e uso de arquiteturas pedagógicas. Simpósio Brasileiro de Informática na Educação (SBIE), 1(1):384-393.

Resende, J., Oliveira, P., Silva, L., Rocha, L., and Albergaria, E. (2019). Librasbot: Um recurso educacional aberto para o estímulo o pensamento lógico de crianças surdas. Simpósio Brasileiro de Informática na Educação (SBIE), 1(1):892-901.

Snoddon, K. (2018). Wfd position paper on inclusive education. https://bit.ly/3ebroWg. Accessed: 2020-06-29.

Tavares, O., Reinoso, L., and Almeida, W. (2017). Cap-apl: plataforma para criação e uso de arquiteturas pedagógicas para aprendizagem de português e libras. Simpósio Brasileiro de Informática na Educação (SBIE), 1(1):466-475.

Vale, V., Mesquita, E., Ferreira, R., Melo, J., and Cintra, S. (2018). O jogo gestus como aplicação de primeiro contato com libras. Anais dos Workshops do Congresso Brasileiro de Informática na Educação (WCBIE), 1(1):8.

WHO (2018). Global estimates on prevalence of hearing loss. https://bit.ly/3g5Of6t. Accessed: 2020-06-29.

Wohlin, C., Runeson, P., Hst, M., Ohlsson, M. C., Regnell, B., and Wessln, A. (2012). Experimentation in Software Engineering. Springer Publishing Company, Incorporated, New York, NY, USA.

Zhang, H., Babar, M. A., and Tell, P. (2011). Identifying relevant studies in software engineering. Information and Software Technology, 53(6):625-637. Special Section: Best papers from the APSEC. 